\title{
Performance of the SoLid reactor neutrino detector
}

\author{
Maja Verstraeten ${ }^{\mathrm{a}}$ (on behalf of the SoLid collaboration) \\ Universiteit Antwerpen, Belgium
}

\begin{abstract}
The SoLid Collaboration is currently operating a 1.6 ton neutrino detector near the Belgian BR2 reactor. Its main goal is the observation of the oscillation of electron antineutrinos to previously undetected flavour states. The highly segmented SoLid detector employs a compound scintillation technology based on PVT scintillator in combination with $\mathrm{LiF}-\mathrm{ZnS}(\mathrm{Ag})$ screens containing the ${ }^{6} \mathrm{Li}$ isotope. The experiment has demonstrated a channel-to-channel response that can be controlled to the level of a few percent, an energy resolution of better than $14 \%$ at $1 \mathrm{MeV}$, and a determination of the interaction vertex with a precision of $5 \mathrm{~cm}$. This contribution highlights the major outcomes of the R\&D program, the quality control during component manufacture and integration, the current performance and stability of the full-scale system, as well as the in-situ calibration of the detector with various radioactive sources.
\end{abstract}

\section{Introduction}

The Standard Model - the rigorous theory of particle physics, incredibly precise and accurate in its predictions - cannot account for the way massive neutrinos show to behave. The neutrino sector is rich in anomalies and little understood phenomena.

The reactor and gallium anomalies are among the most striking and persistent anomalies [1]. The observed antineutrino rate coming from nuclear reactors shows a clear deficit, in comparison to recent calculations [2]. This reactor antineutrino anomaly (RAA), has been observed by multiple collaborations and over a wide range of distances between detector and reactor core. Along with other observed deficits, notably the gallium and LSND/MicroBooNE anomalies [3,4] this combines to a significant deficit.

The deficits can be resolved by introduction of an additional neutrino mass state [5]. Since measurements of the $\mathrm{Z}$ boson production cross section precisely indicate the number of light neutrino species to be three [6], this new neutrino is expected not to interact with ordinary matter through the weak interaction, making it "sterile". The anomalous results suggest squared neutrino mass splittings on the order of $1 \mathrm{eV}^{2}$ and a small mixing angle [2]. Figure 1 shows the parameter space where the RAA is most significant.

A fraction of the anti electron neutrinos that emerge from the reactor core and cross the detector, will interact in the detector volume. The sterile hypothesis argues that neutrinos can oscillate into the sterile state and escape detection altogether, explaining the deficit in detection rate. The eV-scale mass splitting implies meter scale oscillation lengths. Investigating the effect requires very short baselines to the reactor core. The SoLid detector is operated as close as $6.4 \mathrm{~m}$ to the reactor core.

By assessing the disappearance pattern of the reactor neutrinos, the existence and characteristics of the sterile

a e-mail: maja.verstraeten@uantwerpen.be neutrino can be pinpointed. The probability that an electron neutrino of energy $E$ is found as such after travelling a distance $L$, could be influenced by oscillation into the sterile state, as indicated by the formula

$$
P_{e e} \sim 1-\sin ^{2}\left(2 \theta_{e e}\right) \sin ^{2}\left(1.267 \Delta m_{14}^{2} L[m] / E[\mathrm{MeV}]\right) .
$$

The oscillation probability is dictated by the properties of the neutrino states, namely the mixing angle $\theta_{e e}$ and squared mass difference $\Delta m_{14}^{2}$.

The oscillation is apparent over distance and energy. Experimental coverage of the electron neutrino rate over a range of $L / E$ requires a good position and energy resolution. SoLid accomplishes this by deploying a highly voxelized detector that consists of $5 \times 5 \times 5 \mathrm{~cm}^{3}$ cubes. For a sterile neutrino with the parameters of the RAA best fit, the SoLid detector is expected to produce a disappearance pattern similar to that seen in Fig. 3 .

Currently, unexpected spectral features around $6 \mathrm{MeV}$ neutrino energy were observed by long baseline reactor experiments using common fuels $\left({ }^{235} \mathrm{U},{ }^{238} \mathrm{U},{ }^{239} \mathrm{Pu}\right.$, ${ }^{241} \mathrm{Pu}$ ), which are correlated with reactor power and fuel composition [7]. The RAA and spectrum distortions stress the neutrino community's need to better understand the reactor neutrino spectrum. The SoLid collaboration designed and built a finely segmented neutrino detector, demonstrating a novel detector technology using hybrid scintillators [8].

\section{SoLid detector at the BR2 reactor}

The SoLid detector is operated near the Belgian reactor 2 (BR2) at the SCK-CEN. BR2 has an uncommon fuel of highly enriched (>90\%), pure ${ }^{235} \mathrm{U}$. This single fuel isotope is of particular interest to asses the spectral distortion. The research reactor is highly suited for a short baseline oscillation search. The twisted core design (Fig. 2) results in a small core diameter $(\approx 0.5 \mathrm{~m})$, ensuring very little position smearing. The detector is positioned on axis, 


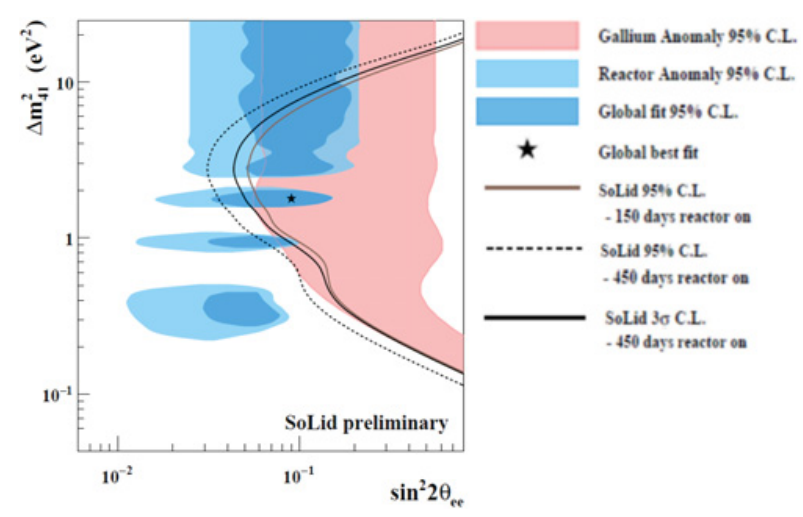

Figure 1. 95\% CL regions of gallium and reactor annomalies are indicated in the $\sin ^{2}\left(2 \theta_{e e}\right)-\Delta m_{41}^{2}$ parameter space. Envisaged contourplot of the SoLid experiment is shown, assuming $30 \%$ IBD efficiency, 14\% energy resolution and S:B of 3.

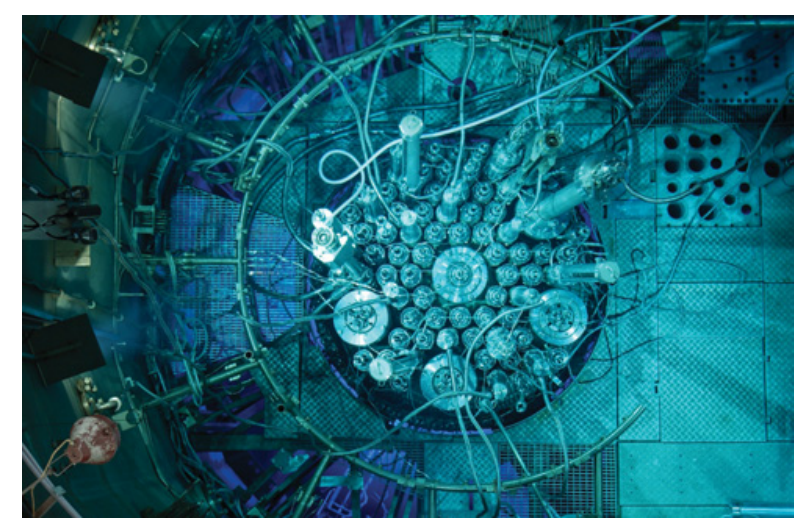

Figure 2. Top view of the fuel matrix of the BR2 tank-inpool reactor core. The twisted design results in a compact core, allowing a detector baseline of $\sim 6 \mathrm{~m}$.

with the smallest distance between detector edge and core center only $6.4 \mathrm{~m}$. We cover a baseline up to $9 \mathrm{~m}$.

The space in the reactor hall is sufficient for a relatively compact, above-ground detector and modest passive shielding. With 10 m.w.e. overburden, the atmospheric backgrounds are challenging. Radioactivity measurements show the intrinsic background is low compared with candidate sites at other reactors. Effective background rejection is performed with active and passive shielding. The complete detector is enclosed in a shipping container which is surrounded by a $50 \mathrm{~cm}$ thick water wall of 28 ton (Fig. 4). The roof structure supports $50 \mathrm{~cm}$ of $\mathrm{HDPE}$ shielding. The 3D topological information obtained by the cubes allows discrimination of the reactor neutrino's signature over the prominent backgrounds.

The reactor is powered about half the year around $60 \mathrm{MW}$, in 1 month cycles. The intermittent reactor off periods allow for an accurate background determination and calibration campaigns. Phase 1 of the experiment, with 1.6 ton active mass, is scheduled to run for 3 years. Efficient signal tagging is required to reach the physics aim.

\section{Neutrino detection principle}

Neutrinos interact with the detector volume via inverse beta decay (IBD), resulting in a positron and a neutron that are correlated in time and space. To optimally detect

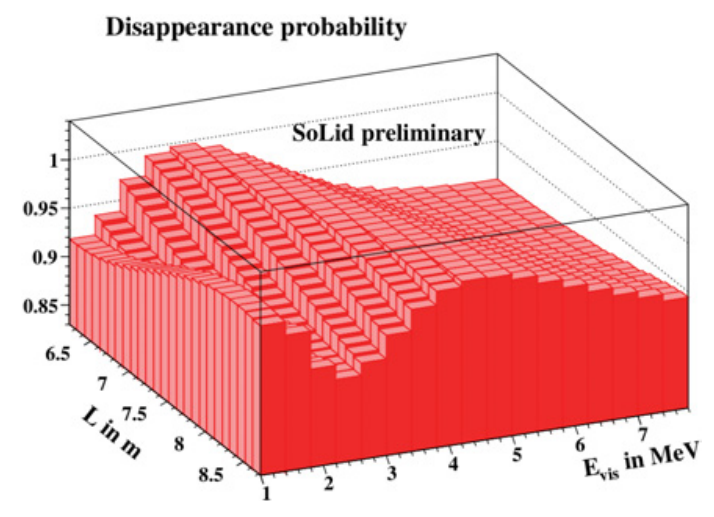

Figure 3. Number of detected IBD events as function of visible energy, $E_{v i s}$, and traveled distance, $L$, in case of oscillations with parameters $\sin ^{2}\left(2 \theta_{e e}\right)=0.09$ and $\Delta m_{41}^{2}=1.78 \mathrm{eV}^{2}$.

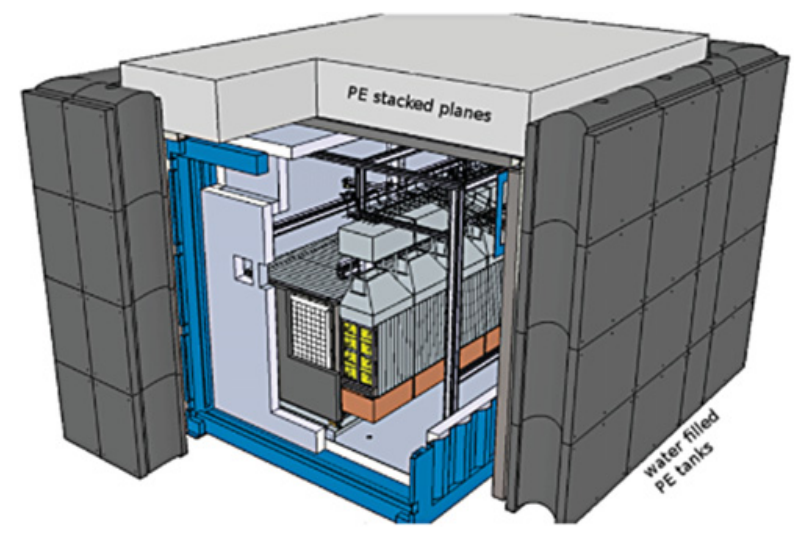

Figure 4. Schematic view of the detector and its passive shielding in Geant4 [9].

and discriminate both particles, two solid scintillators are joined [10]. Cubes of Polyvinyl-toluene (PVT) act as a scintillator for the positron prompt signal (see Fig. 6). PVT offers a high light output and a linear energy response, from which both the location and the energy of the neutrino interaction can be determined.

Sheets of ${ }^{6} \mathrm{LiF}: \mathrm{ZnS}(\mathrm{Ag})$ are placed on two faces of each cube to detect the neutron [10]. After thermalization, the neutron can be captured by a ${ }^{6} \mathrm{Li}$ nucleus. This reaction produces an alpha and a tritium nucleus, sharing $4.78 \mathrm{MeV}$ of kinetic energy. Both are highly ionizing and deposit all their energy within the sheet, scintillating in the $\mathrm{ZnS}(\mathrm{Ag})$ microcrystals. Crucially, this scintillation timescale is considerably slower, at $\mathrm{O}(1) \mu \mathrm{s}$, than all other scintillation signals, at $\mathrm{O}(1) \mathrm{ns}$. With a sampling of $40 \mathrm{MHz}$, Pulse shape discrimination (PSD) can identify and discriminate the signals with high efficiency and purity. The positron and neutron signals are separated 2 cubes or less in $90 \%$ of IBD interactions, and have a time separation of $60 \mu \mathrm{s}$ on average.

Each cell of a PVT cube with two ${ }^{6} \mathrm{LiF}: \mathrm{ZnS}(\mathrm{Ag})$ sheets is wrapped in reflective Tyvek for optical insulation [11]. The cells are arranged in 50 planes of 16 by 16 cells, split in five modules of ten planes each. The scintillation light is guided from the cells towards sensors by an orthogonal grid of wavelength shifting fibers. One end of the fiber is coupled to a mirror, whilst the other is connected to a second generation Hamamatsu silicon photomultiplier. The 3200 readout channels result in an enormous data 


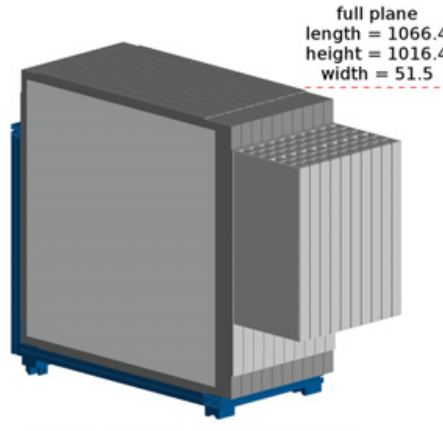

Phasel module $=10$ full planes

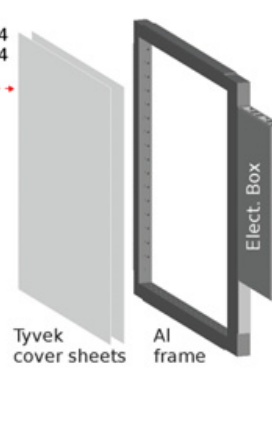

frame
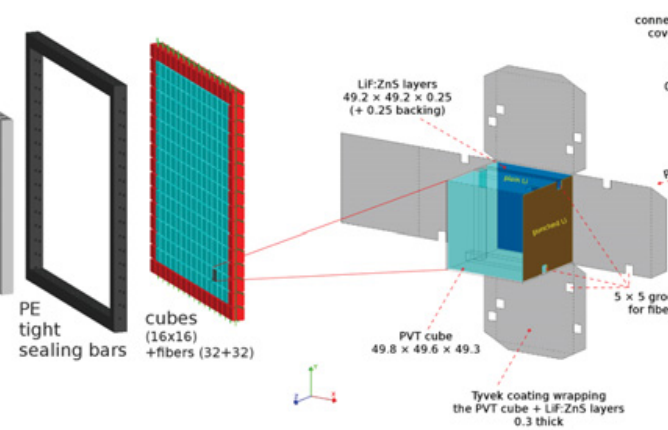

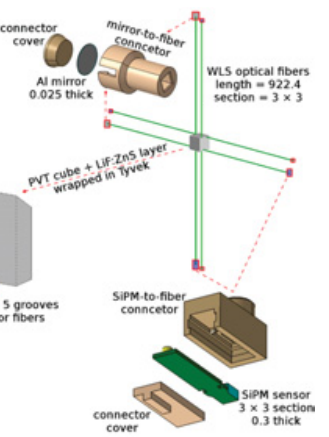

Figure 5. Diagram of a detector module, an exploded frame, cube assembly and fibre readout. Indicated sizes are in $\mathrm{mm}$.

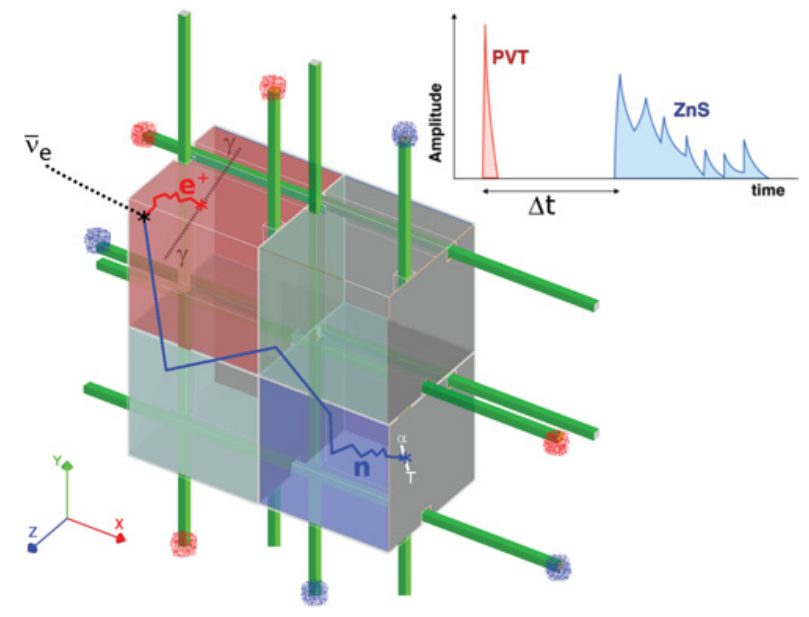

Figure 6. Principle of $\bar{v}_{e}$ detection in cells of combined scintillators. wavelength shifting fibers placed in perpendicular orientations collect the scintillation light.

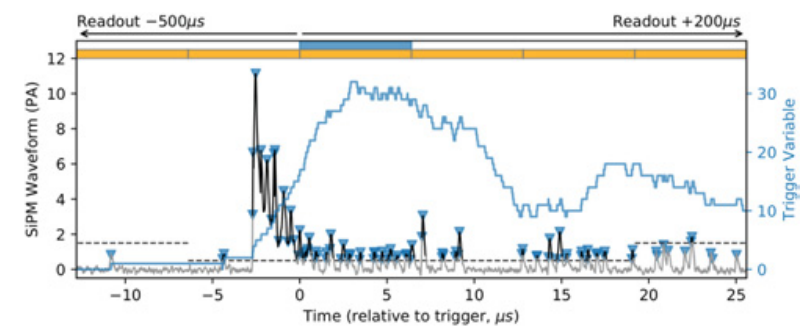

Figure 7. Example neutron waveform (black). The value of the neutron trigger variable (i.e number of peaks in the rolling time window) is shown by the blue line.

rate of $3 \mathrm{~Tb} / \mathrm{s}$. It is handled by a combination of zero suppression and novel triggers, including a dedicated PSD trigger for neutron-like signals [12].

\section{Trigger scheme}

The trigger strategy for the IBD interaction relies on triggering for the resulting neutron [12]. The neutron signals have to be efficiently identified at a sustainable rate. This is feasible since the $\mathrm{ZnS}(\mathrm{Ag})$ signal is characterised by a set of sporadic pulses (as shown in Fig. 4), while the electronic scintillation from PVT consists of one sharp peak.

The algorithm offering best neutron discrimination involves, for each detector channel, counting the peaks of the waveform that are over threshold in a local, rolling timewindow. The algorithm triggers when the number of peaks in the window exceeds a treshold. Upon triggering an IBD buffer is readout, containing the triggered plane, along with its three neighbouring planes on either side, during a time span of $700 \mu \mathrm{s}$. The readout region recorded is considerably larger than the expected extent of the IBD interaction in both space and time. This allows the positron interactions to be recorded without any trigger bias. A scan of the trigger efficiency versus trigger purity was performed as a function of the two trigger parameters - the threshold criteria of the peaks, and the number of required peaks in the rolling time window - at two different over voltages. The optimal trigger settings that were deduced, result in a PSD algorithm for neutron signals that is $\sim 80 \%$ efficient.

To identify high energy signals, an amplitude threshold trigger is implemented, which requires a coincidence of $2 \mathrm{MeV}$ signals on a horizontal and a vertical fiber. The threshold trigger can be used to discriminate backgrounds from signal, such as tagging muons that enter the detector.

The mean recorded data rate is $21 \mathrm{MB} / \mathrm{s}$ during physics mode, which is dominated by the IBD buffer of the neutron trigger, and corresponds to around $1.6 \mathrm{~TB} /$ day.

\section{Signal identification}

The nuclear signal reconstruction is continued by an offline analysis of the recorded data. By selecting the four channels that contain the maximal number of peaks over threshold in the trigger window, the cube position where the interaction took place can be reconstructed. Combining information from these fibers, the amplitude and integral of the signal is computed. The Amplitude versus Integral over Amplitude parameter space is shown in Fig. 8. An efficient and pure separation of electromagnetic signals (ES) and nuclear signals (NS) is visible. The main ES contamination in NS candidates is from high amplitude signals, most likely muons crossing the plane.

\section{Quality assurance}

To validate the necessary performance of the SoLid detector and to identify defective components, a quality assurance process (QA) was developed during construction [13]. An automated calibration system called CALIPSO was constructed according to the diagram in Fig. 9. CALIPSO performed a calibration of each plane before its 


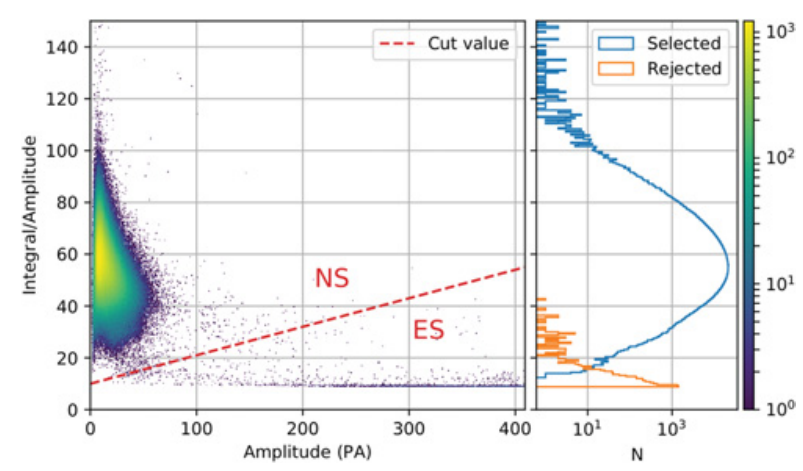

Figure 8. Integral over Amplitude versus Amplitude, in PixelAvalanches (PA) for reconstructed NS candidates from data taken with a ${ }^{252} \mathrm{Cf}$ source. The red dashed line shows the cut used for Particle Identification. Right: projection on the Integral over Amplitude axis for selected and rejected events.

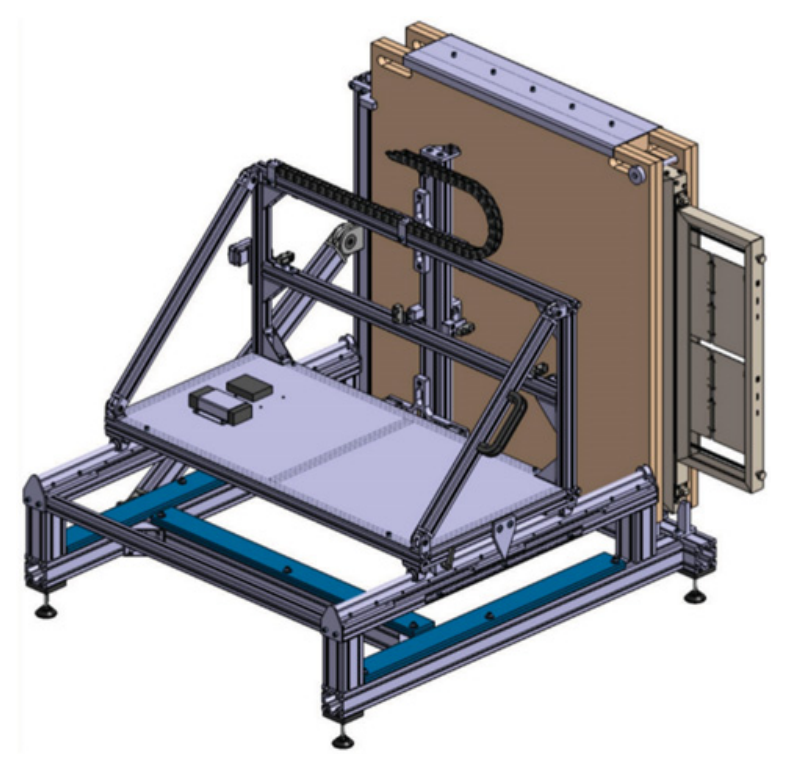

Figure 9. Diagram of the CALIPSO system for calibration of the SoLid planes, which operates in neutron and gamma mode.

integration in the detector. With sub-millimeter precision in the XY axes, the robot can place gamma and neutron radioactive sources in front of each detector cell.

With a ${ }^{22} \mathrm{Na}$ source, a first estimate of the light yield per cube was obtained by measuring the Compton edge of the $1.27 \mathrm{MeV}$ gamma. As shown in Fig. 10, an average light yield of $83 \mathrm{PA} / \mathrm{MeV}$ was reached (without MPPC cross-talk subtraction, which is estimated to be 17\%) [13].

With $\mathrm{AmBe}$ and ${ }^{252} \mathrm{Cf}$ sources, preliminary results for the neutron detection efficiency per plane were obtained and validated, where $\epsilon_{\text {detection }}=\epsilon_{\text {capture }} \times \epsilon_{\text {reconstruction }}$. Figure 10 shows a relative neutron detection efficiency with $5 \%$ dispersion. By comparing with Monte Carlo, the absolute neutron reconstruction efficiency was determined to be $\sim 68.7 \%$ overall [13].

The calibrations show that a good homogeneity is achieved over all detector planes. During QA, minor construction defects were identified and fixed. After passing the QA, the modules were mounted, deployed and commissioned at BR2, which was completed in February 2018.

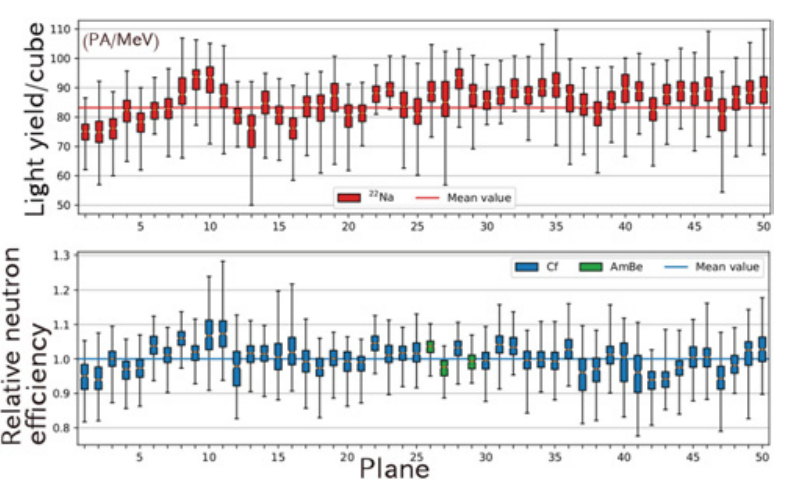

Figure 10. CANDLE plots for 50 planes showing light yield and relative neutron efficiency. Red candles are measured with ${ }^{22} \mathrm{Na}$, blue candles with ${ }^{252} \mathrm{Cf}$ and green with AmBe. Filled boxes represent the plane's cubes between the first and third quartiles. Black lines represent cubes below and above those.

\section{In-situ calibration}

To periodically perform calibration of the detector, in situ a second automated calibration robot is installed on a rail system above the SoLid detector, called CROSS. Between every two modules a gap can be opened (Fig. 12), where CROSS can freely manoeuvre a calibration source.

The homogeneous response of the segmented detector is monitored to be stable. The lightyield is higher than expected with more than 70 pixel avalanches per $\mathrm{MeV}$ deposited. The homogenous neutron reconstruction efficiency is above $75 \%$. A very good agreement was found between the $1.27 \mathrm{MeV}\left({ }^{22} \mathrm{Na}\right)$ and the $4.4 \mathrm{MeV}(\mathrm{AmBe})$ gammas, confirming a linear energy response of the PVT [14]. Further calibrations will be done with a complete set of gamma sources $\left({ }^{137} \mathrm{Cs},{ }^{22} \mathrm{Na},{ }^{60} \mathrm{Co},{ }^{207} \mathrm{Bi}\right.$, and $\left.\mathrm{AmBe}\right)$ to test the energy response in a wider range.

In addition, thanks to the detector's segmentation, muon tracks can be accurately reconstructed. This enables monitoring the detector response on a daily basis and studying the detector response at high energy. Figure 11 left, shows the pressure corrected muon rate during reactor on and off periods in December 2017. The rate is stable over the reactor transition, demonstrating that muons are reconstructed correctly. The typical constant energy deposit of muons is observed clearly, allowing monitoring the PVT energy response. This gives an extra handle for calibration. The right side of Fig. 11 shows a trending plot of muon energy deposits.

\section{Online monitoring}

Since Spring 2018, SoLid is in highly stable data taking mode, for both reactor on and off periods. Run control operations are controlled via a dedicated python based web application. The appliction also provides an interface to monitor SoLid's Data Quality (SDQM). You can oversee data runs, by the fraction that is processed online using SoLid's reconstruction and analysis program (Saffron2), and watch physics variables, that are periodically measured with several environmental sensors. This set of measurements serves as input to an automated alarm system. In case stable data taking is obstructed, alerts are prompted to contact persons.

Long term trends of trigger rates and SiPM measurements are shown in Fig. 13. The transition 

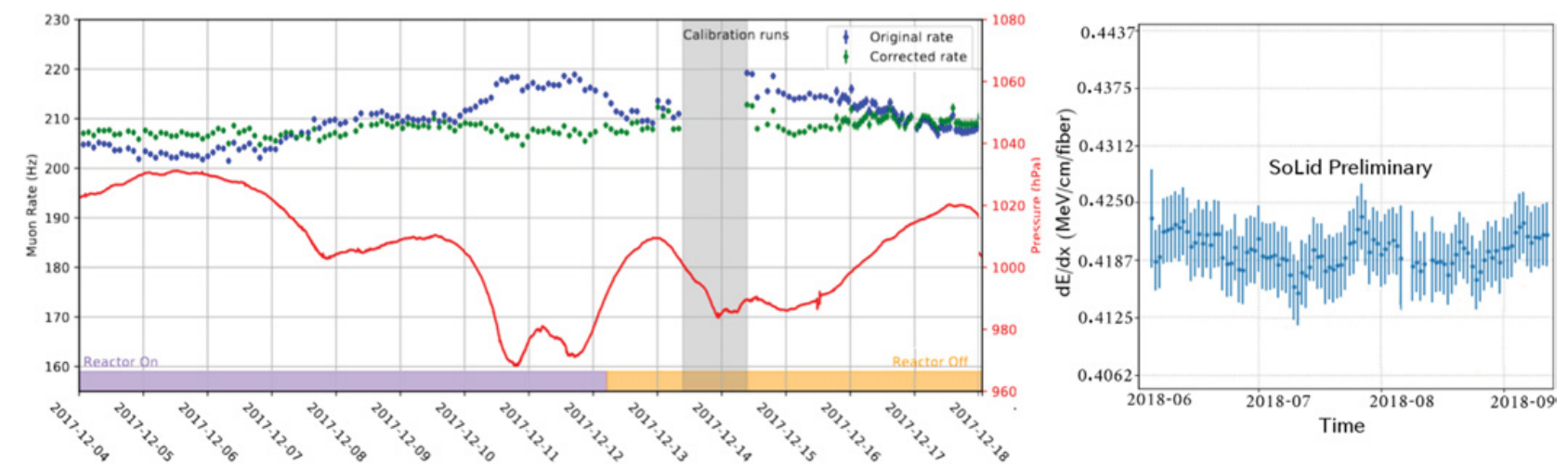

Figure 11. Left: Monitoring of the muon rate during reactor on and off periods, demonstrating accurate muon reconstruction. The correction for pressure variations is shown in green. Right: Monitoring of muon energy deposits.

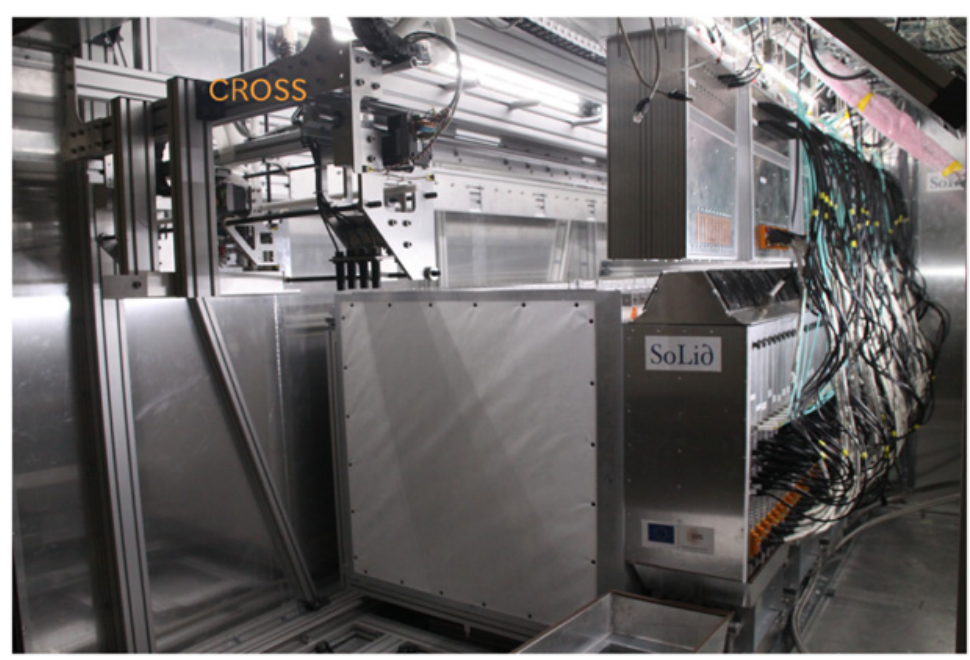

Figure 12. The detector, installed in the container. On the right, the module's service box, electronics and heat exchanger. The automated calibration robot CROSS can lower calibration sources between any modules.

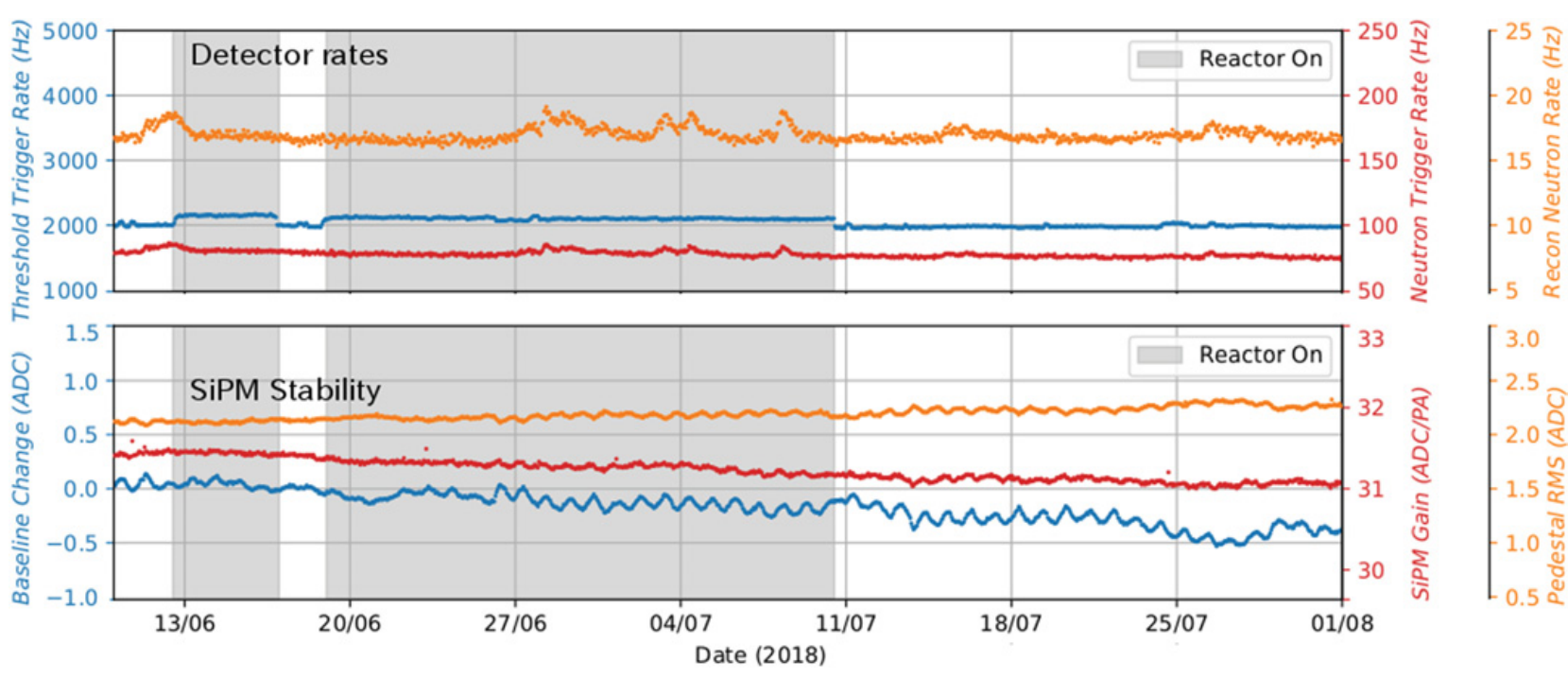

Figure 13. Weekly trends of various detector metrics. Only he threshold trigger rate is sensitive to the reactor on-off transition. 
between reactor on and off can be seen in the small changes in the threshold trigger rate. While the reactor is on, the deviation of the neutron and threshold trigger rates are $2 \%$ and $1 \%$ respectively over a 1 hour period.

The SiPM measurements show small changes over the long term, as well as day-night variations. These changes are correlated with temperature changes up to $0.5^{\circ} \mathrm{C}$ inside the detector container. The variations observed in these metrics are all $<2 \%$, and are not correlated with changes in the trigger rates.

\section{Data taking}

With ongoing data taking, preliminary rate monitoring is performed. The rate of accidentals is determined in an off time window, by making inverted coincidences between ES signals following NS signals, where all other IBD selection criteria are applied. An average rate of only $33.5 \pm 1.4$ events per day is observed for reactor on and $14.5 \pm 1.4$ for reactor off, during May 2018. An excess of around 300 events per day during reactor on is observed [15]. For IBD like events, the time difference between prompt and delayed signal is consistent with the thermalisation and capture of the neutron and the spatial separation is as expected.

The analysis is being further developed. Ultimately, the target sensitivity entails an energy resolution of $14 \%$, an IBD efficiency of $30 \%$ and a signal over background of 3 [8]. Under this assumption, the parameter space concerning the RAA covered by SoLid is indicated in Fig. 1 for cumulative periods of reactor on data taking.

\section{Conclusion}

SoLid successfully deployed a new detector technology. A 1.6 ton detector was commissioned end of 2017. The performance was validated with calibration and commissioning data. Operation is smooth and remote shifts are simplified to the minimum. Automatic calibration with radioactive sources provides precision data for assessing the reactor antineutrino anomaly and to provide a reference measurement of the neutrino spectrum. SoLid is taking good quality physics data and observes IBD-like events. The analyis is further developed. Backgrounds are measured and IBD selection criteria optimized.

\section{References}

[1] M. Dentler, Á. Hernández-Cabezudo, J. Kopp, P.A.N. Machado, M. Maltoni, I. Martinez-Soler, T. Schwetz, J. High Energy Phys. 08, 10 (2018)

[2] G. Mention, M. Fechner, T. Lasserre, D. Mueller, T. Lhuillier, Phys. Rev. D 83, 19 (2011)

[3] A. Aguilar et al., Phys. Rev. D 64, 71 (2001)

[4] A. Aguilar-Arevalo et al., Phys. Rev. Lett. 110, 5 (2013)

[5] M. Dentler, Á. Hernández-Cabezudo, J. Kopp, M. Maltoni, T. Schwetz, J. High Energy Phys. 11, 99 (2017)

[6] D. Groom et al., Eur. Phys. J. C 15, 4 (2000)

[7] P. Huber, Phys. Rev. Lett. 118, 042502 (2017)

[8] Y. Abreu et al., J. Instrum. 12, P04024 (2017)

[9] S. Agostinelli et al. (GEANT4), Nucl. Instrum. Meth. A 506, 250 (2003)

[10] Y. Abreu et al., J. Instrum. 13, P05005 (2018)

[11] Y. Abreu et al., J. Instrum. 13, P09005 (2018)

[12] Y. Abreu et al., JINST 14, P11003 (2019)

[13] Y. Abreu et al., JINST 14(2), P02014 (2019)

[14] L. Manzanillas, Proceedings of Science, p. 4 (2018), arXiv:1811.05694

[15] N. Van Remortel, Talk at XXVIII International Conference on Neutrino Physics and Astrophysics, 4-9 June 2018, Heidelberg, Germany (2018), http://doi.org/10.5281/zenodo. 1287945 